\title{
Dorsal Pancreatic Artery-a Study of Its Detailed Anatomy for Safe Pancreaticoduodenectomy
}

\author{
T Tatsuoka $^{1} \cdot$ T Noie $^{2}$ (D) $\cdot$ T Noro $^{1} \cdot$ M Nakata $^{3} \cdot$ H Yamada $^{4} \cdot$ Y Harihara $^{2}$
}

Received: 29 October 2019 / Accepted: 24 April 2020 / Published online: 15 May 2020

(C) The Author(s) 2020

\begin{abstract}
Early division of the dorsal pancreatic artery (DPA) or its branches to the uncinate process during pancreaticoduodenectomy (PD) in addition to early division of the gastroduodenal artery and inferior pancreaticoduodenal artery should be performed to reduce blood loss by completely avoiding venous congestion. However, the significance of early division of DPA or its branches to the uncinate process has not been reported. The aim of this study was to investigate the anatomy of DPA and its branches to the uncinate process using the currently available high-resolution dynamic computed tomography (CT) as the first step to investigate the significance of DPA in the artery-first approach during PD. Preoperative dynamic thin-slice CT data of 160 consecutive patients who underwent hepato-pancreato-biliary surgery were examined focusing on the anatomy of DPA and its branches to the uncinate process. DPA was recognized in 103 patients (64\%); it originated from the celiac axis or its branches in 70 patients and from the superior mesenteric artery or its branches in 34 patients. The branches to the uncinate process were visualized in 82 patients ( $80 \%$ of those with DPA), with diameters of $0.5-1.5 \mathrm{~mm}$ in approximately $80 \%$ of the 82 patients irrespective of DPA origin. DPA branches to the uncinate process were recognized using high-resolution CT in approximately half of the patients.
\end{abstract}

Keywords Dorsal pancreatic artery $\cdot$ Pancreaticoduodenectomy $\cdot$ Uncinate process

\section{Introduction}

Blood loss reduction by avoiding venous congestion in the resected specimen during pancreaticoduodenectomy (PD) is one of the main indications of the artery-first approach $[3,6,8$, 9, 11, 18, 19, 23]. Early division of the inferior pancreaticoduodenal artery (IPDA) along with the gastroduodenal artery is considered important for avoiding venous congestion using the artery-first approach $[3,6,8,9,11,18,19,23]$.

The dorsal pancreatic artery (DPA) exists in many cases [1, $15,16,24,27]$. DPA ramification is complex, and there are

T Noie

tnoie@east.ntt.co.jp

1 Department of Surgery, Dokkyo Medical University Saitama Medical Center, Saitama, Japan

2 Department of Surgery, NTT Medical Center Tokyo, Tokyo, Japan

3 Department of Radiology, Dokkyo Medical University Saitama Medical Center, Saitama, Japan

4 Department of Radiology, NTT Medical Center Tokyo, Tokyo, Japan many individual differences in DPA anatomy. DPA branches that feed the uncinate process of the pancreas exist in many cases $[1,15,16,24,27]$. In such cases, to completely avoid venous congestion, DPA or its branches distributed to the uncinate process should be divided before dissecting from the portal vein (PV) and superior mesenteric vein (SMV) in the artery-first approach during PD. However, no study has investigated the significance of DPA in the artery-first approach during PD.

Preoperatively, in each case, the surgeon comprehends the pancreatic vascular anatomy necessary for PD from preoperative computed tomography (CT) data. Thus, many studies have reported the CT depiction of the peripancreatic vascular system, including DPA $[4,7$, $10,14,20,22]$. However, CT data of DPA branch distributed to the uncinate process have not been previously reported, although classical dissection studies have reported the anatomy of the uncinate process branch of DPA $[16,27]$. Thus, the aim of the present study was to investigate the anatomy of DPA and its branches to the uncinate process on current high-resolution dynamic CT, as the first step to investigate the significance of DPA in the artery-first approach during PD. 


\section{Materials and Methods}

We assessed preoperative, dynamic, thin-slice CT data of 160 consecutive patients who underwent hepato-pancreato-biliary surgery between January 2016 and December 2017 at our institutions. There were 100 male and 60 female patients with a median age of 70 years (36-87). The diagnoses are pancreatic cancer in 47 patients, bile duct cancer in 23 , intraductal papillary mucinous neoplasm in 22 , and so on.

CT scans were performed using a 320-row multidetector CT scanner (320-MDCT; Aquilion ONE; Toshiba Medical Systems, Tokyo, Japan at Dokkyo Medical University Saitama Medical Center, Japan, and Aquilion ONE; Toshiba Medical Systems, Otawara, Japan at NTT Medical Center Tokyo, Japan). A nonionic contrast agent $(350 \mathrm{mgI} / \mathrm{ml}$, Iomeron; Bracco Eisai Co., Ltd., Tokyo, Japan) was infused at $600 \mathrm{mgI} / \mathrm{kg}$ for $30 \mathrm{~s}$ prior to imaging. Early and late arterial phase images were obtained at 25 and $40 \mathrm{~s}$, respectively, after starting the infusion.

The existence, origin, and ramification and anatomy of DPA, especially the existence of branches to the uncinate process, along with the caliber of the arteries that feed the pancreatic head and existence and origin of the accessory middle colic artery (AMCA) were examined. All imaging data were evaluated by two authors (TT and TN) using a diagnostic workstation (Synapse EX version 1.9; FUJIFILM Medical, Tokyo, Japan) with consensus on readings.

The institutional review boards of the Dokkyo Medical University Saitama Medical Center, Japan, and the NTT Medical Center Tokyo, Japan, approved this retrospective study, and the requirement for informed consent was waived.

\section{Results}

Among the 160 patients, DPA was recognized using preoperative dynamic thin-slice CT in 103 patients (64\%) (Table 1). DPA originated from the celiac axis (CA) or its branches in 70 patients $(68 \%)$ and the superior mesenteric artery (SMA) or its branches in 34 patients (33\%) (one patient had two DPAs originating from both CA and SMA series) (Table 1).

The most representative ramification and the anatomy of DPA originating from CA or its branches is as follows: DPA runs caudally behind the pancreatic neck or body and the proximal part of the splenic vein (SV); rarely initially has a branch to the pancreatic head; sometimes subsequently has a branch to the pancreatic body entering from the cranial side of $\mathrm{SV}$; most often subsequently has branches to the pancreatic body entering from the caudal side of SV (usually connecting to the inferior pancreatic artery); in many cases, finally descends at the left posterior side of SMV and at the right anterior side of SMA; and enters into the uncinate process (Fig. 1a). The origin of DPA from CA or its branches and the frequency of the representative branches are presented in

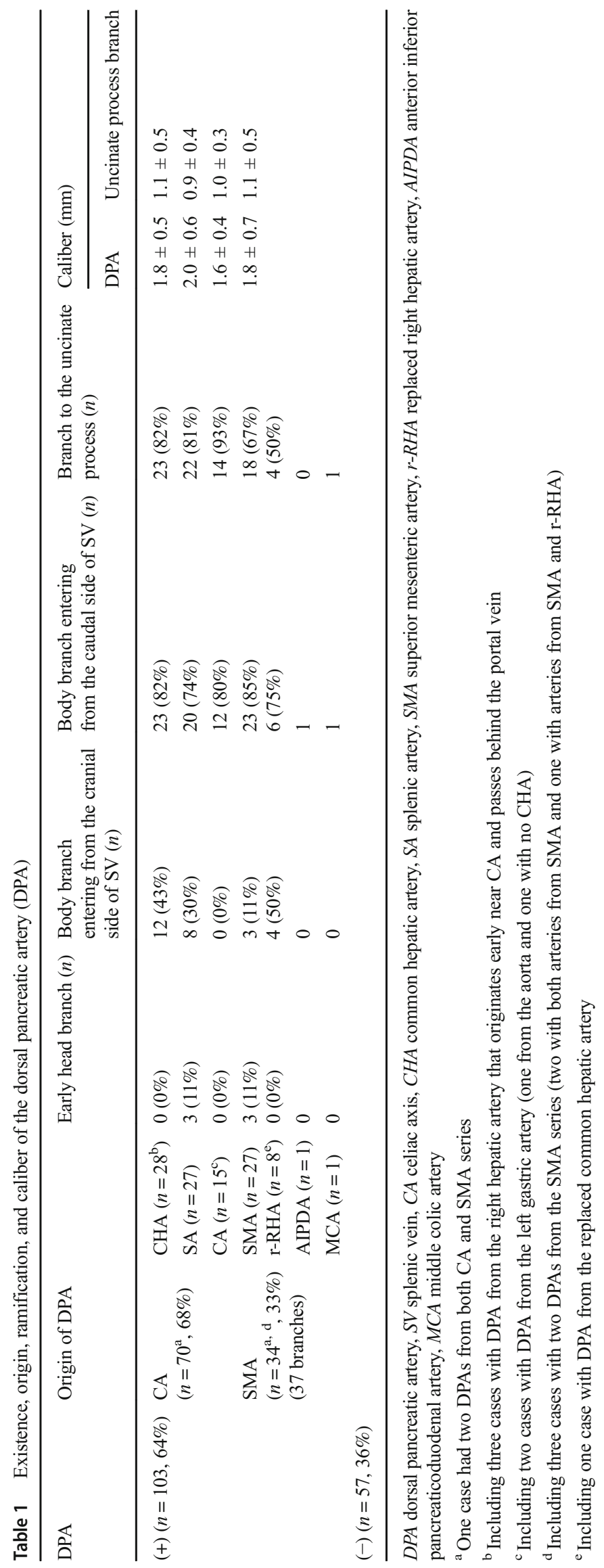




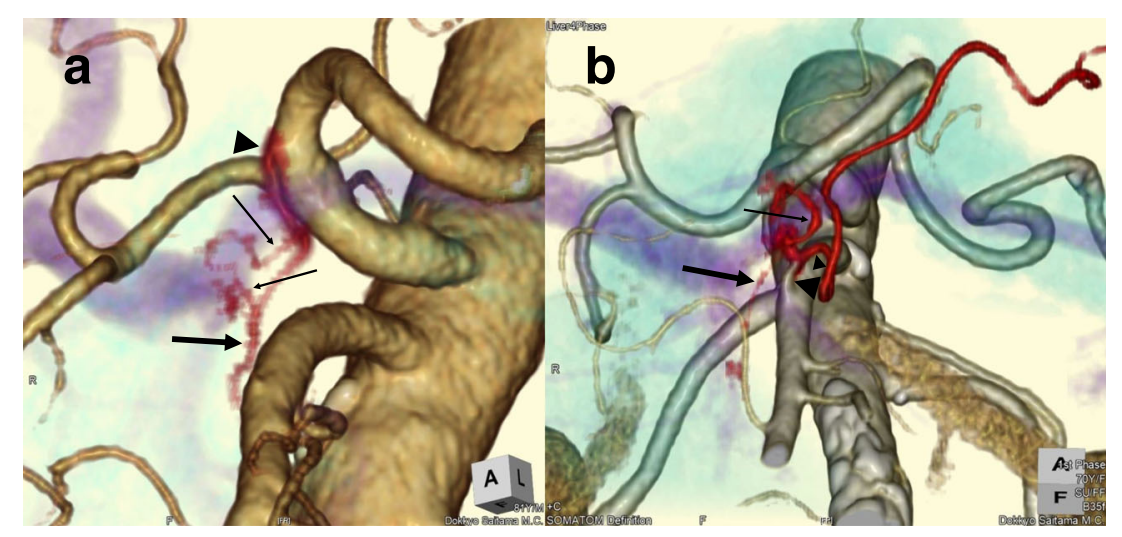

Fig. $13 \mathrm{D}$ reconstruction of the course of the dorsal pancreatic artery. a Dorsal pancreatic artery from the splenic artery. 3D reconstruction of the CT scans of an 81-year-old man with hepatocellular carcinoma. The dorsal pancreatic artery and its branches are depicted as red vessels. The splenic vein (SV) and portal vein are indicated as purple vessels, and the pancreatic parenchyma is indicated in weak blue color. The dorsal pancreatic artery (arrowhead) originates from the splenic artery, runs caudally behind the proximal part of SV, produces branches to the pancreatic body (thin arrow), descends at the right anterior side of the superior mesenteric artery as a branch to the uncinate process (bold arrow), and enters into the uncinate process. b Dorsal pancreatic artery from the

Table 1. The branch to the uncinate process is recognized frequently irrespective of the origin of DPA.

DPA originated from SMA at $15.2 \pm 9.8 \mathrm{~mm}$ caudally from the origin of SMA and proximally than IPDA or the trunk of IPDA and the first jejunal artery (1st JA), except in one case, in which DPA originated from the SMA between the origins of IPDA and 1st JA. DPA usually arises from the anterior or right anterior wall of SMA, anteriorly ascends right for several millimeters, and then divides into branches to the pancreatic body and uncinate process (Fig. 1b). The origin of DPA from SMA or its branches and the frequency of the representative branches are presented in Table 1. The branch to the uncinate process from DPA originating from SMA or its branches was less frequently recognized (but not significantly) than that from DPA originating from CA or its branches (23/37 vs. $59 / 70)$. The overall incidence of the branch to the uncinate process was $80 \%(82 / 103)$.

The prepancreatic arterial arcade (see "Discussion") was recognized in only three (3\%) among the 103 patients with DPA. Additionally, in five cases, a thin branch, which originated from the branch to the pancreatic body entering from the caudal side of SV, ran caudally from the left to right in front of SMV at the level of the caudal edge of the pancreatic neck; however, it disappeared at the right of SMV, and prepancreatic arterial arcade could not be identified.

The calibers of DPA and the branch to the uncinate process are illustrated in Tables 1 and 2. The caliber of the branch to the uncinate process rangede from $0.2 \mathrm{~mm}$ to approximately $2 \mathrm{~mm}$. Irrespective of the origin of DPA, the mean caliber was approximately $1 \mathrm{~mm}$, and the calibers were between $0.5 \mathrm{~mm}$ and $1.5 \mathrm{~mm}$ in approximately $80 \%$ of the patients. The mean superior mesenteric artery. 3D reconstruction of the CT scans of a 70year-old woman with gallbladder cancer. The dorsal pancreatic artery and its branches are depicted as red vessels. The splenic vein and portal vein are depicted as purple vessels, and the pancreatic parenchyma is depicted in weak blue color. The dorsal pancreatic artery (large arrowhead) originates from the superior mesenteric artery (SMA), ascends in front of SMA, initially produces the accessory middle colic artery (small arrowhead) in this case, and divides into branches to the pancreatic body (thin arrow) and uncinate process (bold arrow). The branch to the uncinate process descends at the right side of SMA and enters into the uncinate process

diameters of the gastroduodenal artery and IPDA were $3.4 \pm$ $0.8 \mathrm{~mm}$ and $1.8 \pm 0.6 \mathrm{~mm}$, respectively.

AMCA arises from SMA slightly more proximally compared with the 1st JA and courses to the upper left to meet the marginal artery at the splenic flexure. Conversely, the middle colic artery originates from SMA a little more distally than the 1st JA. AMCA was observed in 32 of the 160 patients $(20 \%)$, and the incidence of AMCA was not different irrespective of the presence or absence of DPA (Table 3). Among 19 patients in whom AMCA was recognized along with DPA, AMCA originated from DPA in nine and from CA in six (Table 4).

\section{Discussion}

The artery-first approach has been advocated by many pancreatic surgeons for the early determination of resectability and curability of advanced pancreatic cancer before performing an irreversible step during $\mathrm{PD}[2,3,5,6,8,9$, $13,17,18,21,23,25,26,28]$. The key point of this technique is dissection between the pancreatic head and SMA early during PD (i.e., the early division of IPDA). In contrast to traditional PD, in which IPDA is usually divided during the last step of resection after dissection from PV and SMV, the early division of IPDA along with that of the gastroduodenal artery helps to avoid venous congestion of the resected specimen and contributes to blood loss reduction during PD.

Therefore, many pancreatic surgeons have routinely adopted the artery-first approach in patients with periampullary diseases other than advanced pancreatic cancers to reduce blood loss during PD $[3,6,8,9,11,18,19$, 
Table 2 Distribution of the caliber of the uncinate process branch

\begin{tabular}{lllll}
\hline Origin of DPA & \multicolumn{2}{l}{ Caliber of uncinate process branch } \\
\cline { 2 - 5 } & $\leq 0.5 \mathrm{~mm}$ & $>0.5 \mathrm{~mm}, \leq 1.0 \mathrm{~mm}$ & $>1.0 \mathrm{~mm}, \leq 1.5 \mathrm{~mm}$ & $>1.5 \mathrm{~mm}$ \\
\hline CHA $(n=23)$ & $2(9 \%)$ & $8(35 \%)$ & $12(52 \%)$ & $1(4 \%)$ \\
SA $(n=22)$ & $3(14 \%)$ & $12(55 \%)$ & $6(27 \%)$ & $1(5 \%)$ \\
CA $(n=14)$ & $2(14 \%)$ & $5(36 \%)$ & $7(50 \%)$ & $0(0 \%)$ \\
SMA $(n=23)$ & $3(13 \%)$ & $7(30 \%)$ & $8(35 \%)$ & $5(22 \%)$ \\
Total $(n=82)$ & $10(12 \%)$ & $32(39 \%)$ & $33(40 \%)$ & $7(9 \%)$ \\
\hline
\end{tabular}

CHA common hepatic artery, including one case with DPA from the right hepatic artery that originates early near CA and passes behind the portal vein, $S A$ splenic artery, $C A$ celiac axis, including one case with DPA from the left gastric artery from the aorta, SMA superior mesenteric artery, including four cases with DPA from the replaced right hepatic artery and one case with DPA from the middle colic artery

23]. Early division of IPDA along with the gastroduodenal artery has been considered important for avoiding venous congestion using the artery-first approach. However, many surgeons may experience the following situation. After dividing IPDA at its origin and dissecting the pancreatic head from SMV and PV, at the last step of resection, one or two thin arteries need to be divided when dissecting the nerve plexus of the pancreatic head. These arteries are the branches to the uncinate process from DPA. Without prior division of these arteries, completely avoiding venous congestion of the resected specimen cannot be achieved. However, no study has investigated the significance of early division of DPA or its branches to the uncinate process in the artery-first approach during PD.

Several problems need to be resolved for investigating the significance of early division of DPA in the artery-first approach during PD. The first problem that needs to be resolved is the anatomy and frequency of the uncinate process branches of DPA, which have many individual differences with regard to anatomy and ramification. The second is examining the technical feasibility of the early division of DPA or its branches to the uncinate process, according to the origin and ramification of DPA. The third is the implementation of a prospective study examining whether early division of DPA or its branches to the uncinate process helps in reducing blood loss during PD.

This study was conducted to elucidate the anatomy and frequency of the uncinate process branches of DPA detected using current high-resolution dynamic CT. The incidence of

Table 3 Accessory middle colic artery

\begin{tabular}{lll}
\hline & \multicolumn{2}{l}{ Accessory middle colic artery } \\
\cline { 2 - 3 } & $(+)$ & $(-)$ \\
\hline Total & $32(20 \%)$ & $128(80 \%)$ \\
DPA (+) & $19(18 \%)$ & $84(82 \%)$ \\
DPA (-) & $13(23 \%)$ & $44(77 \%)$ \\
\hline
\end{tabular}

$D P A$ dorsal pancreatic artery
DPA has been reported to range from $64 \%$ to $100 \%$ in anatomical and radiological studies $[1,4,7,10,14-16,20,22,24$, 27]. According to Michels [16], DPA is characterized by a course that crosses behind the proximal part of SV. However, the pancreatic magna artery, a large superior pancreatic branch of the splenic artery, may be erroneously recognized as DPA, especially in angiographical studies. This may explain the reported previously high incidence of DPA. The incidence of DPA (64\%) identified in the present study corresponds to the minimum incidence previously reported.

Woodburne and Olsen [27] and Michels [16] reported in their dissection studies that DPA typically has two right branches and a left branch. This left branch corresponds to the branch entering the body in the present study. In their reports, a right branch, after producing the uncinate branch, turns across the pancreatic head to form a prepancreatic arterial arcade with a small left branch of the anterior superior pancreaticoduodenal artery. In the present study, this

Table 4 Accessory middle colic artery (AMCA) from the dorsal pancreatic artery (DPA)

\begin{tabular}{lll}
\hline DPA origin & AMCA from DPA & $\begin{array}{l}\text { AMCA from SMA } \\
\text { (not from DPA) }\end{array}$ \\
\hline CHA $(n=28)^{\mathrm{a}}$ & 3 & 2 \\
$\mathrm{SA}(n=27)^{\mathrm{b}}$ & 3 & 3 \\
$\mathrm{CA}(n=15)^{\mathrm{c}}$ & 0 & 4 \\
SMA $(n=24)^{\mathrm{d}}$ & 3 & 0 \\
r-RHA $(n=8)^{\mathrm{e}}$ & 0 & 1 \\
Total & 9 & 10
\end{tabular}

${ }^{\text {a }}$ CHA common hepatic artery, including three cases with DPA from the right hepatic artery that originates early near $\mathrm{CA}$ and passes behind the portal vein

${ }^{\mathrm{b}} S A$ splenic artery

${ }^{\mathrm{c}} C A$ celiac axis, including two cases with DPA from the left gastric artery (one from the aorta and one with no CHA)

${ }^{\mathrm{d}}$ SMA superior mesenteric artery; 24 cases with 27 DPAs

${ }^{\mathrm{e}} r$-RHA replaced right hepatic artery, including one case with DPA from the replaced common hepatic artery 
prepancreatic arterial arcade was recognized in three cases; however, the branch forming the prepancreatic arterial arcade originated from the branch to the pancreatic body. The same anatomical thin branch was recognized in five cases additionally, but the prepancreatic arterial arcade could not be identified. This difference may be associated with the small caliber of this artery or with the limited resolution capacity of the current fine CT scans, which is affected by imaging timing. In cases with the prepancreatic arterial arcade, this branch can be easily divided in front of SMV early during PD.

Few studies have reported the frequency of individual branches of DPA [15, 16, 24, 27]. Woodburne and Olsen [27] reported that a prepancreatic arterial arcade had an incidence of $93.3 \%$ among 90\% DPA occurrences. In a dissection study, Matsumura [15] showed that the uncinate branch had an incidence of $60.9 \%$ among $88.8 \%$ DPA occurrences. The $80 \%$ incidence of the branch to the uncinate process in our study is between these values.

The caliber of DPA branch to the uncinate process has never been reported. In the present study, the caliber ranged from $0.2 \mathrm{~mm}$ to approximately $2 \mathrm{~mm}$ and was between 0.5 and $1.5 \mathrm{~mm}$ in approximately $80 \%$ of the patients. It is known that a large caliber is associated with a strong influence on venous congestion. The significance of early division of DPA or its branches to the uncinate process will differ according to the caliber.

The feasibility of the early division of DPA or its branches to the uncinate process depends on the extent of lymph node dissection necessary along with the origin and ramification of DPA. For example, in a case in which DPA originates from SMA, the preceding division of the branch from DPA to the uncinate process with preservation of the branches to the pancreatic body is not very difficult using the supracolic anterior approach [8]. Additionally, in a case in which DPA originates from the splenic artery, the early division of DPA at its origin and preceding division of the branches from DPA to the pancreatic body are practically possible in a patient with pancreatic head cancer. It is noteworthy that venous congestion cannot be completely avoided without dividing branches from DPA to the pancreatic body before dissection from SMV and PV, even after dividing DPA at its origin. However, this technique is not practical for a patient with low-grade malignancy. To ascertain the significance of early division of DPA or its branches to the uncinate process in the artery-first approach during PD, a prospective study is required. However, the possible frequency of early division of DPA or its branches to the uncinate process according to disease variety and DPA anatomy should be examined before such a prospective study.

AMCA, which is also designated as the superior left colic artery [12], should be kept in mind as one of the anomalies concerning DPA, although it has little significance in the artery-first approach during PD.
To sum up. using the currently available high-resolution dynamic CT, DPA was identified in $64 \%$ of total patients included in the study, and branches to the uncinate process were visualized in $80 \%$ of those with DPA. In approximately $80 \%$ of the patients, the calibers of the branches to the uncinate process from DPA are between 0.5 and $1.5 \mathrm{~mm}$ irrespective of DPA origin.

Acknowledgments The study received no funding. The authors would like to thank Enago (www.enago.jp) for the English language review.

Presented in part at the 73th General Meeting of the Japanese Society of Gastroenterological Surgery, Kagoshima, Japan, July 2018.

Author Contribution T Tatsuoka: Data collection, Manuscript writing.

T Noie: Project development, Data collections and management, Data analysis, Manuscript writing/editing.

T Noro: Project development, Critically revising manuscript.

M Nakata: Data collection, Manuscript writing.

H Yamada: Data collection, Manuscript writing.

Y Harihara: Project development, Critically revising manuscript.

\section{Compliance with Ethical Standards}

Conflicts of Interest The authors declare that they have no conflict of interest.

Ethical Approval All procedures performed in studies involving human participants were in accordance with the ethical standards of the institutional and/or national research committee and with the 1964 Helsinki declaration and its later amendments or comparable ethical standards.

Informed Consent The requirement for informed consent was waived by the institutional review boards of Dokkyo Medical University Saitama Medical Center, Japan, and the NTT Medical Center Tokyo, Japan.

Open Access This article is licensed under a Creative Commons Attribution 4.0 International License, which permits use, sharing, adaptation, distribution and reproduction in any medium or format, as long as you give appropriate credit to the original author(s) and the source, provide a link to the Creative Commons licence, and indicate if changes were made. The images or other third party material in this article are included in the article's Creative Commons licence, unless indicated otherwise in a credit line to the material. If material is not included in the article's Creative Commons licence and your intended use is not permitted by statutory regulation or exceeds the permitted use, you will need to obtain permission directly from the copyright holder. To view a copy of this licence, visit http://creativecommons.org/licenses/by/4.0/.

\section{References}

1. Bartelli E, Gregorio FD, Mosca S, Bastianini A (1998) The arterial blood supply of the pancreas: a review. V. the dorsal pancreatic artery. An anatomical review and a radiological study. Surg Radiol Anat 20:445-452

2. Chiaro MD, Segersvard R, Rangelova E et al (2015) CattellBraasch maneuver combined with artery-first approach for superior mesenteric-portal vein resection during pancreatectomy. J Gastrointest Surg 19:2264-2268 
3. Dumitrascu T, David L, Popescu I (2010) Posterior versus standard approach in pancreatoduodenectomy: a case-match study. Langenbeck's Arch Surg 395:677-684

4. Fang CH, Kong D, Wang X, Wang H, Xiang N, Fan Y, Yang J, Zhong SZ (2014) Three-dimensional reconstruction of the peripancreatic vascular system based on computed tomographic angiography images and its clinical application in the surgical management of pancreatic tumors. Pancreas 43:389-395

5. Hackert T, Werner J, Weitz J, Schmidt J, Buchler MW (2010) Uncinate process first-a novel approach for pancreatic head resection. Langenbeck's Arch Surg 395:1161-1164

6. Horiguchi A, Ishihara S, Ito M, Nagata H, Shimizu T, Furusawa K, Kato R, Katada K, Miyakawa S (2007) Pancreatoduodenectomy in which dissection of the efferent arteries of the head of the pancreas is performed first. J Hepato-Biliary-Pancreat Surg 14:575-578

7. Horiguchi A, Ishihara S, Ito M, Nagata H, Asano Y, Yamamoto T, Kato R, Katada K, Miyakawa S (2008) Multislice CT study of pancreatic head arterial dominance. J Hepato-Biliary-Pancreat Surg 15:322-326

8. Inoue Y, Saiura A, Yoshioka R, Ono Y, Takahashi M, Arita J, Takahashi Y, Koga R (2015) Pancreatoduodenectomy with systematic mesopancreas dissection using a supracolic anterior artery-first approach. Ann Surg 262:1092-1101

9. Ironside N, Barreto SG, Loveday B, Shrikhande SV, Windsor JA, Pandanaboyana S (2018) Meta-analysis of an artery-first approach versus standard pancreatoduodenectomy on perioperative outcomes and survival. Br J Surg 105:628-636

10. Ishigaki S, Itoh S, Satake H, Ota T, Ishigaki T (2007) CT depiction of small arteries in the pancreatic head: assessment using coronal reformatted images with 16-channel multislice CT. Abdom Imaging 32:215-223

11. Ishizaki Y, Sugo H, Yoshimoto J, Imamura H, Kawasaki S (2010) Pancreatoduodenectomy with or without early ligation of the inferior pancreatoduodenal artery: comparison of intraoperative blood loss and short-term outcome. World J Surg 34:2939-2344

12. Koizumi M, Horiguchi M (1990) Accessory arteries supplying the human transverse colon. Acta Anat 137:246-251

13. Kurosaki I, Minagawa M, Takano K, Takizawa K, Hatakeyama K (2011) Left posterior approach to the superior mesenteric vascular pedicle in pancreaticoduodenectomy for cancer of the pancreatic head. JOP 12:220-229

14. Lin Y, Yang X, Chen Z, Tan J, Zhong Q, Yang L, Wu Z (2012) Demonstration of the dorsal pancreatic artery by CTA to facilitate superselective arterial infusion of stem cells into the pancreas. Eur $\mathrm{J}$ Radiol 81:461-465

15. Matsumura H (1998) The significance of the morphology of the dorsal pancreatic artery in determining the presence of the accessory right hepatic artery passing behind the portal vein. Acta Anat Nippon 73:517-527
16. Michels NA (1962) The anatomic variations of the arterial pancreaticoduodenal arcades: their import in regional resection involving the gallbladder, bile ducts, liver, pancreas and parts of the small and large intestines. J Inter Coll Surg 37:13-40

17. Nakao A, Takagi H (1993) Isolated pancreatectomy for pancreatic head carcinoma using catheter bypass of the portal vein. Hepatogastroenterology 40:426-429

18. Negoi I, Hostiuc S, Runcanu A, Negoi RI, Beuran M (2017) Superior mesenteric artery first approach versus standard pancreaticoduodenectomy: a systematic review and meta-analysis. Hepatobiliary Pancreat Dis Int 16:127-138

19. Ohigashi H, Ishikawa O, Eguchi H, Yamada T, Sasaki Y, Noura S, Takachi K, Miyashiro I, Murata K, Doki Y, Imaoka S (2004) Early ligation of the inferior pancreaticodudenal artery to reduce blood loss during pancreaticoduodenectomy. Hepatogastroenterology 51: 4-5

20. Okahara M, Mori H, Kiyosue H, Yamada Y, Sagara Y, Matsumoto S (2010) Arterial supply to the pancreas: variations and crosssectional anatomy. Abdom Imaging 35:134-142

21. Pessaux P, Varma D, Arnaud JP (2006) Pancreaticoduodenectomy: superior mesenteric artery first approach. J Gastrointest Surg 10: 607-611

22. Sakaguchi T, Suzuki S, Inaba K, Takehara Y, Nasu H, Konno H (2012) Peripancreatic arterial anatomy analyzed by 3D multidetector-row computed tomography. Hepatogastroenterology 59:1986-1989

23. Sanjay P, Takaori K, Govil S, Shrikhande SV, Windsor JA (2012) 'Artery-first' approaches to pancreatoduodenectomy. Br J Surg 99: $1027-1035$

24. Tsusumi M, Arakawa T, Terashima T et al (2014) Morphological analysis of the branches of the dorsal pancreatic artery and their clinical significance. Clin Anat 27:645-652

25. Varty PP, Yamamoto H, Farges O, Belghiti J, Sauvanet A (2005) Early retropancreatic dissection during pancreaticoduodenectomy. Am J Surg 189:488-491

26. Weitz J, Rahbari N, Koch M, Buchler MW (2010) The "artery first" approach for resection of pancreas head cancer. J Am Coll Surg 210:e1-e4

27. Woodburne RT, Olsen LL (1951) The arteries of the pancreas. Anat Rec 111:255-270

28. Zhu J, Han D, Li X et al (2016) Inferior infracolic 'superior mesenteric artery first' approach with a no-touch isolation surgical technique in patients with a borderline resectable cancer of the pancreatic head. Ann Surg Oncol 23:S976-S980

Publisher's Note Springer Nature remains neutral with regard to jurisdictional claims in published maps and institutional affiliations. 